\title{
Pick My Dress: Beacon based Navigation System to find your Suitable Wear
}

\author{
M. J. E. Fernando \\ Student of Sri Lanka \\ Institute of Information \\ Technology (SLIIT)
}

\author{
N. M. Thenuwara \\ Student of Sri Lanka \\ Institute of Information \\ Technology (SLIIT)
}

\author{
S. S. Shurane \\ Student of Sri Lanka \\ Institute of Information \\ Technology (SLIIT)
}

\author{
L. S. Wijesinghe \\ Student of Sri Lanka \\ Institute of Information \\ Technology (SLIIT)
}

\author{
D. Dhammearatchi \\ Lecturerat Sri Lanka \\ Institute of Information \\ Technology (SLIIT)
}

\begin{abstract}
Retailing has become a major part of busy current lifestyle when it comes to every single event of people's lives. When considering retail purposes clothing shops, shopping malls and retail boulevards come into the play. Usually it is a vast area with lots of people, shops, goods and paths which makes the area looks more and more complex. Since the time has become the major barrier of their lives, people are willing to have everything done at their fingertips more quickly and without any hard attempt. In a vastarea of single clothing shop customers face difficulties in finding their preferable items in a certain time. Though it is a new shop or a well-known shop which he/she usually visits, with the busy time period they are already dealing with makes the situation unnecessarily complex and confusing. Correct paths to the preferred item are sometimes hard to find because of the immensecrowd and area which anyway give them a hard time. There are several difficulties customers are experiencing when matching the clothes they are trying to buy, these difficulties are briefly explained in this document. For the people who are already in a war with their time, do not want to spend their precious time and effort to get an idea about the similar items or matching items to the item they selected, 'Pick My Dress' application will let peoples to get over from those issues. In other hand, clothing shop owners can get more benefits out of this application to promote their shops all over the country. Having a mobile application which will do all the shopping, matching dresses and navigation facility to find dress items in one single screen, helps shop owners to get more attraction of customers. To move forward with modern developing technology, cloths shop owners can use this new application which will bring cloth shopping into a new different level. The system uses BLE (Bluetooth Low Energy) for its implementation process with the aid of Beacons and stickers. Therefore the 'Pick My Dress' mobile application has been able to answer all those current issues mentioned above by helping the customers in their retailing purposes.
\end{abstract}

\section{Keywords}

Beacons, stickers, Bluetooth, BLE, navigation, Retailing, shopping, Data mining

\section{INTRODUCTION}

For the customers with their busy life styles, it is difficult to waste their time on shopping, especially in buying clothes. They have to spend more time on finding the path to the particular item they are looking forward to buy. After finding a particular item the customer might have to travel elsewhere maybe to a place they already journeyed to find another item.

The main objective of this research is to make the customers' tasks easy and give them a best service through an android based application with indoor navigation. This research will help customers to select most preferable clothes from the Android mobile application and they can easily navigate to the place where the clothes are and also they can find the ideal matches to the selected clothes. When they are passing the suggested matching clothes they will be notified by a popup message.

In building the indoor navigation system in the mobile application, the Bluetooth connection of the mobile devices and Bluetooth signals of the beacons and stickers are being used. Sensitivity of one beacon is more than $20 \mathrm{~m}$ distance and sensitivity of a sticker is about $5 \mathrm{~m}$. In creating this application mainly Bluetooth technology is used. Furthermore, by using a data mining algorithm suggestions related to selected products are being recommended. Beacons are small devices that transmit a low-power signal that can be picked up by nearby Bluetooth-enabled mobile devices/smart phones, and they are widely used for many purposes. New arrival of beacon 'stickers' assists in getting information about particular item separately, while beacons are supporting in the general Bluetooth connection to mobile devices.

The user can use his mobile phone and connect to the system by enabling the Bluetooth connection of the mobile phone. Meanwhile the user must enable the Wi-Fi (Wireless Fidelity) connection of the mobile and through Wi-Fi connection the mobile will be automatically connected to the clothing shop's database through a web server. Database provides all the suggestions with the help of the mobile application. Site map is used in giving the indoor navigation facility.

From 'Pick My Dress' application's indoor navigation process, customers can easily find the path to the preferred place and the auto dress suggestion system can save their time. Since this is a user friendly simple application users will be able to easily use this system.

This research paper consists of design and implementation details of an Android application to navigate inside a clothing shop. The authors have several objectives as follows:

- Providing navigation through Beacons and Beacon Stickers.

- To provide dress suggestions to the selected clothes by gender. 
- To process data efficiently.

- To develop a successful real time notification system.

The rest of this paper is organized as follows. Section 2 has provided the existing related work. Section 3 describes the methodology of this paper. The results and discussion is mentioned in Section 4, and the conclusion of the Pick My Dress project has described in Section 5.

\section{LITERATURE VIEW}

Vidanapathirana et.al states that the major capability of the 'HospiX' android application is finding location or providing navigation directions to the users while they are at hospital premises. HospiX application has a limitation of not able to use it in IOS platform since it is implemented only for Android users. Though this is used in hospitals, $24 \times 7$ crowded places like shopping stores does not have this technology to use it for the ease of customers' lives. In order to cover that area 'Pick My Dress' application provided accurate navigations inside the shopping store with the aid of Beacons and Beacon Stickers which provides directions to relevant dresses which customer prefers most [1].

As Pierlot and Droogenbroeck mention, this application presents a new active beacon-based angle measurement system used for mobile robot positioning with several major innovations.Overall BeAMS system is small, flexible, low power and tractable solution used for robot positioning. Though they used rotating sensors to catch infrared signals more accurately, there is a disadvantage in transmitting information and power to the sensors if these are located on the turning part of the system. As in 'Pick My Dress' application there are beacons used to transmit signals for the Bluetooth connection with mobile devices [2].

As Patil and Sankalp states the concept of Beacon Stations and how they help to find various band opening conditions are mainly focused in this system. Also with the use of Beacon station one can find proper band as well as get guideline for Navigation. However the uncertainty in propagation band causes lots of difficulties in operation. Rather than using a beacon station in a one particular place it can be used in a wide area for better purposes. Focusing on that point 'Pick My Dress' application provided indoor navigations for retail shops by using several beacons which can be easily connected with Bluetooth to mobile devices [3].

Alcala-fdez et.al states that KEEL1 (Knowledge Extraction based on Evolutionary Learning) toll is an open source software which supports data management and a designer of experiments through data mining. There are mainly five rule learning methodologies used to carry out this study which are; Ant-Miner, CO-Evolutionary Rule Extractor (CORE), Hierarchical Decision Rules (HIDER), Steady-State Genetic Algorithm for Extracting Fuzzy Classification Rules From Data (SGERD) and Tree Analysis with Randomly Generated and Evolved Trees (TARGET) methodology [4].

Jingjing Yang et.al has introduced a Beacon based indoor positioning system for patients to find their wards in hospitals. The location of users can be reflected by Beacons. To calculate the shortest path between each two Beacons the shortest distance algorithm, Floyd has used. Though this is used in hospitals, crowded places like shopping stores need some advanced technology for the ease of customers'. In order to satisfy that need "Pick My Dress" application hopes to provide accurate navigations inside the shopping store with
Beacons and Beacon Stickers by providing most preferable matching clothes to the customer as suggestions [5].

Rung-Shiang Cheng et.al mentions that with the emergence of Location-Based Services (LBS), the need to locate the position of the user by BLE beacons with a high degree of accuracy has emerged as an important concern. The design of this system includes "storage data design", "positioning method design", "algorithm design" and "graphic design". In order to cover the area of indoor navigation also, "Pick My Dress" application is created with the use of Beacons and Beacon Stickers [6].

Homayounfar, P. and Owoc, M.L. states that there is a major impact from Data Mining techniques on Computerized Patient Record (CPR) systems. The CPR contains medical data, laboratory data and organizational data from different sources with the purpose of patients care. The shown characteristics of medical and organizational data in CPR lead to the special problem of analyzing and linking data from different sources and qualities together. Where in the "Pick My Dress" application,data mining can bridge the important gap and bring together the essence of the information. This will allow to get accurate information through the mobile application [7].

Divya Tomar and Sonali Agarwal states that Data Mining is becoming popular in healthcare field because there is a need of efficient analytical methodology for detecting unknown and valuable information in health data. In making efficient healthcare policies, constructing drug recommendation systems, developing health profiles of individuals, detecting frauds in health insurance, detecting causes of diseases and identifying medical treatment methods data mining techniques can be very useful. Concerning about that, the use of Data Mining techniques provides benefit to the implemented mobile application "Pick My Dress" by providing accurate suggestions to the selected item [8].

\section{METHODOLOGY}

The major mobile application is running in android platform which has the main capability of providing navigation to the users while they are at the particular clothing store. This has been done by beacons, which uses Bluetooth 4.0 connectivity. It is a small, low-energy device that can be installed or positioned almost anywhere. It broadcasts short data strings to all the nearby portable electronic devices including smartphones, tablets. These type of devices perform action when it is close to proximity of a beacon. The new arrival of beacon 'stickers' assists in getting information about particular item separately, while beacons are supporting in the general Bluetooth connection to mobile devices. Mainly application have a navigation interface to give directions to the selected cloth and a suggestion interface to provide ideal matches to the selected item as suggestions. Suggestions for selected item are giving through a data mining algorithm.

Project has selected to use the Prototype method as the development methodology because this model can classify the level or phase of development carefully so that the smoothness of the project can be improved.

In the planning phase research team has aimed to identify the business value, scope and boundaries of the problem, plan goals, strategies for system simulation, analyze feasibility and develop the work plan.

In analysis phase mainly delivers the information about users of the system, when the system will be used, information gathering, process modeling and data modeling through a 
system proposal. This mobile application will be used among customers of a particular retail shop who are capable of handling a smart phone with Bluetooth technology. Implemented application will be used inside a clothing shop when customer wants to find the exact location of a selected garment. Data for the data mining process was gathered from a particular company with a special permission as a formal request. When the needed data is gathered as required, the process was held step by step accordingly. Data will be modeled through decision tree data mining algorithm which is performed by SQL Server Business Intelligence Development Studio 2008. Then the process modeling was started by designing navigation paths according to the floor plan of the particular cloth shop. Coding of the Beacons and the mobile application was created afterwards through Android Studio version 1.5.1.

The main activity in design phase was the development design for various components of the application architecture and interface architecture and determining specifically how the application will work. Basic design of the project was created according to the overview diagram shown in figure 1 . Site plan includes indoor map of the shop where user connects from his/her mobile device through Bluetooth connection. User connects to the database which is in the same Wi-Fi network through the web server using JSON (JavaScript Object Notation).

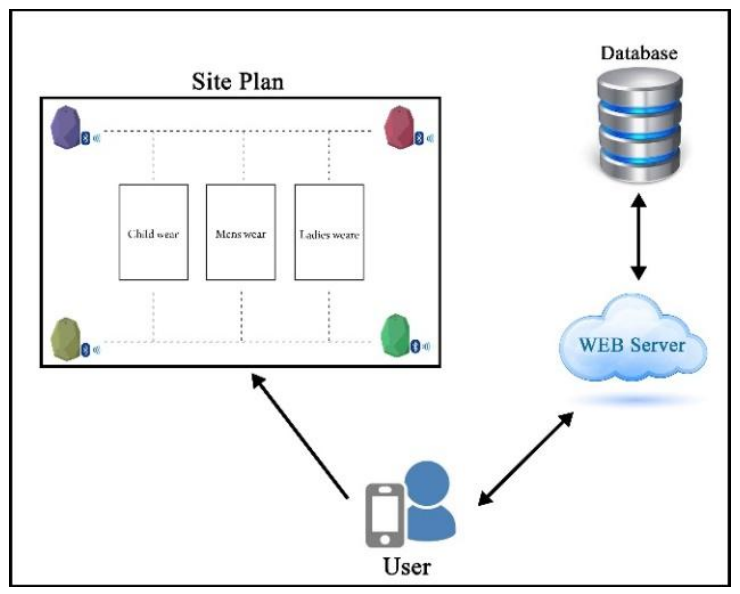

Figure 1: Overview Diagram

The structure of the application was built together with the navigation interface and the suggestion interface mainly. The navigation interface is visible to the customer on their mobile phones which is connected to the particular shop through Bluetooth connection. Basic inputs such as name, gender and age are taking at the main interface. Then the next interface will provide available items in the shop, which will manually be selected as customer's choice. When customer selects the particular item then they have to select the brand that they need. Then the navigation path will be shown with a suggestion interface which will provide some matching clothing items to the particular selected item. When the customer is done with the prior selected wear, he can select any suggested product and view the navigation path to that product also.

In the system beacons are used to find the current location of the user. If the user is in range of a beacon developer can get the distance from proximity value that gives by beacon. Change of proximity values can get the current location of the user. The locations of the stickers are saved in a database table. Each sticker has assigned to a particular brand. When the customer selects that particular brand beacon identify the current location and give the path to selected item. When customer passes each sticker it gives a notification to the customer that they pass this secession.

All the cloth types are saved in a two separate list as male and female. When the customer selects their gender it loads the particular list as the Category interface. When the customer select a category system check the database what are the brands that available for this category. Then it loads results to sub-category interface. This process has done with PHP (Personal Home Page) and JSON. After customer has selected a cloth it checks the highest previous searcher item with that item and give it as suggestions to the customer.

Once the development was successfully completed, the team moved on to testing phase. The research team used unit testing to check the behavior and the response of each and every separate unit developed in the development phase. When the well performances of those units are undoubtedly confirmed, the integration testing was started. The modules were integrated one by one and in each time it was tested independently. Once the integration was completed with the testing, the team took the system as a whole module and tested it to check how the system works as a whole and whether it meets the requirements that planned.

\section{RESULTS AND DISCUSSION}

\subsection{Results}

All the way through from this research the primary finding that has been found was the possibility of using Beacons to find any location in a limited area. With the help of the Beacons and Beacons stickers the team has been able to developed indoor navigation system which runs on Android platform. Developing an android application which connected to the beacons in order to get the navigations of desire location, gave the research team a chance to work with android platform.

Besides the application, data mining model that was developed to make the predictions as a part of the Pick My Dress application is another finding that the team came across with this project. Following Figures represents the main interfaces of this Pick My Dress Application.

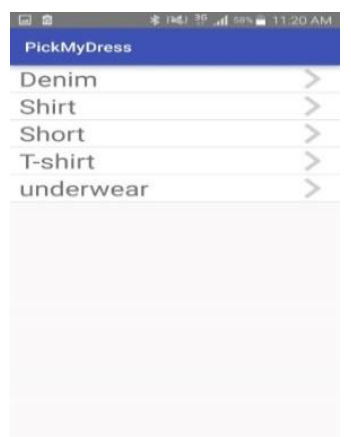

Figure 2: Main Category Selector 




Figure 3: Navigation Plan

Figure 2 shows the available main categories for male customers at the shop database. User can select one main category and navigate into the next interface same as the Figure 2, which gives user the chance to select the sub category or the brand of the prior selected cloth. The floor navigations of the selected sub category will be given to the user with the interface shown in the figure 3.

Following code segment showed in Figure 4 represents the database connection between the shop database and mobile application.



Figure 4: Connection between database and mobile application

Code segments shown in Figure 5 and Figure 6 represent the JSON response and the connection between the server and the mobile application accordingly.

\section{2 \$json_response=json_encode( $\$$ response); 63 echo \$json_response;}

Figure 5: JSON Response

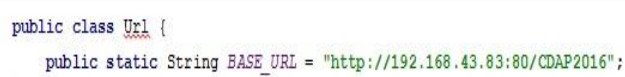

Figure 6: Connection between server and mobile application

The accuracy chart which is a result of data mining section is shown below in Figure 7.

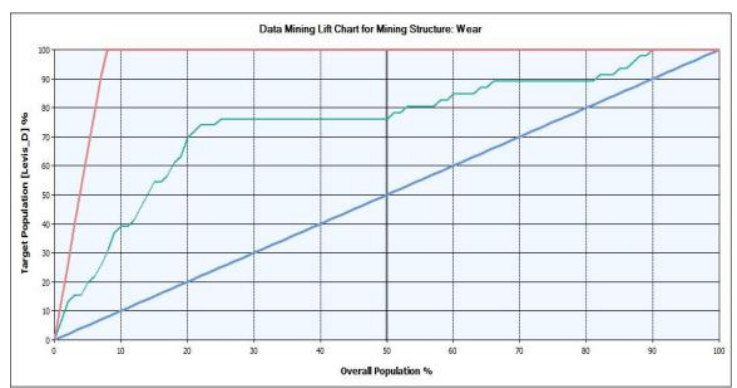

Figure 7: Accuracy chart

\subsection{Discussion}

When considering about navigation technology, most of the existing applications use Wi-Fi and GPS technology commonly. But due to the situation of the 'Pick My Dress' mobile application, increment of the number of users can be a limitation when considering about those earlier considered technologies. Though Wi-Fi and GPS technology is accurate for larger areas, it is not that much useful for small geographic areas like clothing shops. This is the main reason why 'Pick My Dress' is developed using Beacons which uses Bluetooth technology.

The use of Beacon is enhanced with the latest technology of Beacon Stickers which help the navigation technology more and more accurate and easy. Since it has the ability of giving the Bluetooth coverage for a considerable area accurately,

'Pick My Dress' application users will be able to find the exact location of their choice inside the clothing shop without wasting their time.

Since the technology of Beacon Sticker is considered as a research component, the team had a technical problem of not detecting the stickers correctly. With the aid of Bluetooth, basic Beacons were able to detect easily without any constraint. When the team informed about the problem of beacon stickers to the Estimote Website which is the company of those Beacons and Beacon stickers, they re-sent an errorfree Beacon sticker pack when they are clear about the problem which the team is currently having.

Since this system is mainly based on the component of suggesting some related products to the prior selected product in the shop, keeping the database updated with the latest selections of customers is very much important. In order to fulfill that situation within the system, the team came up with an in-built learning system to the database newly, with the request of the lecturer-in-charge. When customer selects two or more products together, that particular purchase information is sent to the database as a record. Through that the team will be able to keep up-to-date information within the shop database which will be helpful in getting related suggestions through the 'Pick My Dress' mobile application.

\section{CONCLUSION AND FUTURE WORK}

Walking in a massive area of a crowded clothing shop to find their preferable cloth can add extra layer of stress and fatigue to a person who is already surrounded with a busy schedule. So an indoor navigation application like Pick My Dress can give them kind of a relief up to an extent where they can easily catch the needed location inside the shop. Additionally they are provided with the service of suggesting related products to a prior selected item which is very much useful for busy customers inside.

The use of Beacons and Beacon Stickers provide an accurate navigation in the customers' mobile device with the use of Bluetooth technology. Catching up the exact location of the cloth item and provided with the location based notifications can be highlighted as the main functions of Pick My Dress mobile application.

Connecting to the Wi-Fi connection of the shop, customer can immediately connected with the database of the particular shop which helps in suggesting related products in the mobile application.

Pick My Dress application currently has a limitation which it can be only used by the users with android devices. Therefore 
this mobile application can be modified in iOS platform also as a next step beyond this research.

\section{ACKNOWLEDGMENT}

We would like to thank for all the guidance given by Mrs.Gayana Fernando as the lecturer in-charge of Comprehensive Design and Analysis Project and to the members of Sri Lanka Institute of Information Technology (SLIIT) for providing adequate resources needed.

Finally, the research team would like to thank all the known and anonymous people who took part in the questionnaire for giving us information which were really important for the development of the project.

\section{REFERENCES}

[1] Vidanapathirana V.S.P., Peiris K.H.M.R., Dhammearatchi D. "HospiX: The Hospital Exploring Application for Smart Devices", International Journal of Scientific and Research Publications, vol. 5-Issue 11, November 2015 [Online]. Available: http://www.slideshare.net/VishviVidanapathiran/hospixthe-hospital-exploring-application-for-smart-devices. [Accessed: 05-Feb-2016]

[2] Pierlot V., Droogenbroeck M.V. "BeAMS: A Beacon based Angle Measurement Sensor for mobile robot positioning", IEEE Transactions on Robotics, vol. 30 Issue 3, pp. 533-549, June 2014 [Online]. Available: http://orbi.ulg.be/bitstream/2268/153647/1/Pierlot2014B eAMS.pdf. [Accessed: 06-Feb-2016]

[3] D. Patil, Dr.S. Sankpal, Electrical and Computational System," International Journal of Electronics and computer systems (IJEECS)", vol. 3, Issue 7, September 2014 [Online]. Available: http://academicscience.co.in/admin/resources/project/pap er/f201409231411468475.pdf/ [Accessed: 05-Feb-2016]
[4] J.Alcala-fdez, A.Fernandez, J.Luengo, J.Derrac, S.Gracia, L.Sanchez and F.Herrera, "KEEL Data-Mining Software Tool: Data Set Repository, Integration of Algorithms and Experimental Analysis Framework", Journal of Multi-Valued Logic \& Soft Computing, vol 17, pp. 255-287, 2011 [Online] Available:http://citeseerx.ist.psu.edu/viewdoc/download? doi=10.1.1.294.9855\&rep=rep1\&type=pdf/ [Accessed: 05-Feb-2016]

[5] J. Yang, Z. Wang and X. Zhang, "An iBeacon-based Indoor Positioning Systems for Hospitals",International Journal of Smart Home, vol. 9, no. 7, pp. 161-168, 2015 [Online]. Available: http://www.sersc.org/journals/IJSH/vol9_no7_2015/16.p df. [Accessed: 07- Feb- 2016]

[6] R. Cheng, W. Hong, J. Wang and K. Lin, "Seamless Guidance System Combining GPS, BLE Beacon, and NFC Technologies", Mobile Information Systems, vol. 2016, pp. 1-12, 2016 [Online]. Available: http://www.hindawi.com/journals/misy/2016/5032365/. [Accessed: 08- Feb- 2016]

[7] P. Homayounfar and M. Owoc, "Data Mining Research Trends in Computerized Patient Records", inThe Federated Conference on Computer Science and Information Systems, 2011, pp. 133-139 [Online]. Available: https://fedcsis.org/proceedings/2011/pliks/142.pdf. [Accessed: 06- Feb- 2016]

[8] D. Tomar and S. Agarwal, "A survey on Data Mining approaches for Healthcare", International Journal of BioScience and Bio-Technology, vol. 5, no. 5, pp. 241-266, 2013 [Online]. Available: http://www.sersc.org/journals/IJBSBT/vol5_no5/25.pdf. [Accessed: 05- Feb- 2016] 